\title{
ATIVIDADE FÍSICA E INCAPACIDADE FUNCIONAL EM IDOSOS DA ZONA RURAL DE UM MUNICÍPIO DO NORDESTE DO BRASIL
}

\author{
Physical activity level and functional disability among elderly \\ in the rural area of a municipality in northeastern Brazil \\ Actividad fisica e incapacidad funcional de mayores de la zona \\ rural de un municipio del Noreste de Brasil
}

Artigo Original

\section{RESUMO}

Objetivo: Analisar a associação entre o nível de atividade física habitual e a incapacidade funcional de idosos residentes em áreas rurais. Métodos: Estudo transversal com 104 idosos residentes em uma zona rural de um município do Nordeste do Brasil. Foram utilizados um questionário com informações sociodemográficas e hábitos de vida, o questionário internacional de atividades físicas (versão adaptada para idosos) e a Escala de Katz para avaliação do estado funcional na realização das atividades básicas da vida diária (ABVD). Para avaliar a associação das variáveis de interesse, foram utilizados como medida de associação a razão de prevalência e seus respectivos intervalos de confiança, e como medida de significância estatística foi utilizado o teste qui-quadrado de Pearson, adotando $\mathrm{p} \leq 0,05$. Resultados: A maioria dos idosos foi classificada como insuficientemente ativa $(64,2 \%)$. Associações a níveis estatisticamente significantes foram observadas entre atividade física e idade ( $\mathrm{p}=0,004)$, situação conjugal $(\mathrm{p}=0,020)$ e escolaridade $(\mathrm{p}=0,002)$. A prática de atividade física associou-se à incapacidade funcional para a realização das $\mathrm{ABVD}(\mathrm{p}=0,017)$. Quando estratificada por sexo e idade, a associação não se manteve $(\mathrm{p}>0,05)$. Conclusão: A prática regular de atividade física apresenta-se como um comportamento importante na prevenção/ melhora da incapacidade funcional entre a população de idosos avaliados.

Descritores: Atividade Física; Idoso; População Rural.

\section{ABSTRACT}

Objective: To analyze the association between the habitual physical activity and functional disability among elderly people living in rural areas. Methods: Cross-sectional study with 104 elderly residents in a rural area of a municipality of northeastern Brazil. The study used a questionnaire with sociodemographic and lifestyle habits information, the International Physical Activity Questionnaire (version adapted for the elderly), and the Katz scale for assessment of functional status in performing the basic activities of daily living (BADL). To assess the association between the variables of interest, the prevalence ratio $(P R)$ and its confidence intervals were used as measure of association, and Pearson's chi-square test was performed as a measure of statistical significance, adopting $p \leq 0.05$. Results: The majority of the elderly were classified as insufficiently active (64.2\%). Associations of statistical significance were observed between physical activity and age $(p=0.004)$, marital status $(p=0.020)$, and education ( $p=0.002)$. The physical activity practice was associated with the functional disability to perform the BADL $(p=0.017)$. When stratified by age and sex, the association was not maintained ( $p>0.05$ ). Conclusion: Regular practice of physical activity is evidenced as an important behaviour in prevention/improvement of functional disability among the assessed elderly population.

Recebido em: 03/09/2014 Revisado em: 05/12/2014 Aceito em: 28/01/2015 


\section{RESUMEN}

Objetivo: Analizar la asociación del nivel de actividad física habitual y la incapacidad funcional de mayores que viven en la zona rural. Métodos: Estudio transversal con 104 mayores de la zona rural de un municipio del Noreste de Brasil. Fueron utilizados un cuestionario con informaciones sociodemograficas y los hábitos de vida, el cuestionario internacional de actividades físicas (versión adaptada a los mayores) y la Escala de Katz para la evaluación del estado funcional en la realización de las actividades básicas de la vida diaria (ABVD). Fueron utilizados la razón de prevalencia y sus respectivos intervalos de confianza para evaluar la asociación de las variables de interés. Para la medición de la significación fue utilizada la prueba de Chicuadrado de Pearson con $p \leq 0,05$. Resultados: La mayoría de los mayores fue clasificada como activos insuficientes (64,2\%). Se observó asociaciones estadísticamente significativas entre la actividad fisica y la edad $(p=0,004)$, la situación conyugal $(p=0,020)$ y escolaridad $(p=0,002)$. La práctica de actividad física se asoció con la incapacidad funcional para la realización de las ABVD $(p=0,017)$. La asociación no se mantuvo $(p>0,05)$ al estratificarla por el sexo y la edad. Conclusión: La práctica regular de actividad física es una conducta importante en la prevención/mejoría de la incapacidad funcional de la población de mayores evaluados.

Descriptores: Actividad motora; Anciano; Población rural.

\section{INTRODUÇÃO}

A mudança no perfil epidemiológico e demográfico ocorrida nas últimas décadas alterou a estrutura etária da população brasileira. A cada ano, 650 mil novos idosos passam a configurar a população brasileira, e a maioria deles encontra-se mais vulnerável a doenças crônicas e a comprometimentos na capacidade funcional ${ }^{(1)}$. Esse cenário contribui para a mudança na relação do cuidado à saúde e maior atenção à população idosa, no que concerne a ações de promoção à saúde para esses indivíduos.

O envelhecimento está diretamente relacionado à maior prevalência de incapacidade e dependência funcional ${ }^{(2,3)}$, estando intimamente associado à redução de massa, força, potência e resistência muscular ${ }^{(4)}$. A incapacidade funcional caracteriza-se como perdas da habilidade de executar atividades que permitem o autocuidado e viver de maneira independente, comprometendo a qualidade de vida e a realização de atividades cotidianas ${ }^{(5)}$.

Além do envelhecimento, o sedentarismo e/ou a prática insuficiente de atividade física apresenta-se como um fator de risco para o declínio da aptidão física (musculoesquelética e cardiorrespiratória) e da capacidade funcional ${ }^{(6,7)}$.
Evidências da literatura demonstram que a prática regular de atividade física é um importante fator de proteção para a incapacidade funcional entre idosos, uma vez que minimiza a degeneração provocada pelo envelhecimento, através do incremento na força muscular, na densidade óssea, no equilíbrio dinâmico e no estado funcional global $^{(8,9,10)}$

Nesse sentido, a atividade física apresenta-se como parte fundamental dos programas mundiais de promoção à saúde, com vistas a garantir um envelhecimento saudável ${ }^{(11)}$. Entretanto, ainda são elevados os índices de inatividade física em todo o mundo, principalmente entre a população $\operatorname{senil}^{(12)}$.

Embora seja crescente o número de estudos direcionados a essa população, são incipientes os levantamentos acerca da associação entre atividade física e incapacidade funcional entre idosos residentes em áreas rurais. Essa população apresenta particularidades, como: menor média de rendimento, níveis educacionais mais inferiores, isolamento geográfico com pouco acesso a transporte público, maior número e mais severos problemas de saúde, serviços de saúde escassos, inacessíveis e mais custosos quando comparados aos disponíveis na zona urbana, tornando-os mais susceptíveis a incapacidades funcionais ${ }^{(13)}$.

Em contrapartida, os idosos da zona rural apresentam vantagens em comparação aos seus pares urbanos, como estilo de vida mais saudável, com menor consumo de tabaco ${ }^{(14,15)}$, maior incorporação do comportamento ativo em atividades do cotidiano (atividades agrícolas, jardinagem, caminhadas e pesca) ${ }^{(16)}$ e hábitos alimentares mais saudáveis. Nesse sentido, o estudo teve como objetivo analisar a associação entre o nível de atividade física habitual e a incapacidade funcional de idosos residentes em áreas rurais.

\section{MÉTODOS}

Estudo de corte transversal, realizado no distrito de Itajuru, zona rural do município de Jequié-BA, no período de agosto a dezembro de 2011. O município de Jequié fica localizado na região sudoeste do estado baiano, com população estimada de 151.820 habitantes ${ }^{(17)}$.

Um censo foi conduzido entre a população de 104 indivíduos com idade igual ou superior a 60 anos, residentes no distrito de Itajuru, que estavam cadastrados na Unidade de Saúde da Família que cobre a zona rural do município.

Foram excluídos aqueles com diagnóstico de demência ou qualquer outro tipo de alteração cognitiva e/ ou diagnóstico ou relato de surdez que inviabilizasse a 
entrevista. A população final do estudo foi composta por 95 indivíduos.

Os dados foram coletados através da aplicação de um questionário estruturado, aplicado em forma de entrevista. Incluíram-se neste estudo as seguintes informações: características sociodemográficas (sexo, idade, situação conjugal, nível de escolaridade e renda mensal); hábitos de vida (etilismo e tabagismo, por meio de questões dicotômicas do tipo sim/não sobre o consumo atual dessas substâncias, sendo o consumo de tais substâncias auto referido pelos idosos); estado funcional (autopercepção do desempenho nas atividades básicas da vida diária - ABVD) e atividade física habitual.

Para a avaliação do estado funcional, utilizou-se a escala de $\mathrm{Katz}^{(18)}$ (reflete os padrões de desenvolvimento no idoso, começando pelas atividades mais complexas, como vestir-se, banhar-se, até chegar às de autorregulação, como alimentar-se, e às de eliminação ou excreção), adotando escores $<5$ como ponto de corte para incapacidade funcional.

Avaliou-se a atividade física habitual pelo Questionário Internacional de Atividade Física (IPAQ) - versão adaptada para idosos ${ }^{(19)}$, instrumento que mede o tempo semanal gasto na realização de atividade física, de intensidade moderada a vigorosa, em diferentes domínios. Como ponto de corte, foram considerados insuficientemente ativos os idosos que, na soma das atividades físicas nos diferentes domínios, exercitaram menos de 150 minutos de atividades moderadas ou vigorosas por semana; e foram considerados ativos os que praticaram acima desse valor ${ }^{(20)}$.

Para avaliar a associação das variáveis de interesse (atividade física e capacidade funcional), foram utilizados como medida de associação a razão de prevalência (RP) e seus respectivos intervalos de confiança (IC), e como medida de significância estatística foi utilizado o teste quiquadrado de Pearson, adotando o nível de significância de $p \leq 0,05$. Os dados foram analisados por meio do programa SPSS 9.0.

O estudo seguiu a Resolução 466/12 do Conselho Nacional de Saúde, sendo a coleta de dados iniciada após aprovação pelo Comitê de Ética em Pesquisa com Seres Humanos da Universidade Estadual do Sudoeste da Bahia (Parecer $n^{\circ}$ 045/2011).

\section{RESULTADOS}

Entre os entrevistados, a média de idade foi de $73,5 \pm$ 9,4 anos. Observou-se um maior percentual de mulheres $(57,9 \% ; n=55)$, indivíduos na faixa etária entre 60 e 79 anos
(75,8\%; $\mathrm{n}=72)$, com baixo nível de escolaridade $(65,3 \%$; $\mathrm{n}=62)$, que vivem sem companheiro $(52,6 \%)$ e com baixos níveis de renda $(96,2 \% ; n=76$ tinham renda de até 1 salário mínimo). No que se refere às características comportamentais, identificou-se que a maioria dos entrevistados não consumia bebidas alcoólicas $(84,2 \% \mathrm{n}=80)$ e não tinha o hábito de fumar $(80,5 \% \mathrm{n}=70)$ (Tabela I).

Tabela I - Características sociodemográficas e hábitos de vida da população idosa residente em zona rural estudada. Itajuru-BA, 2011.

\begin{tabular}{lcc}
\hline \multirow{2}{*}{ Variável } & \multicolumn{2}{c}{ Frequências } \\
\cline { 2 - 3 } & $\mathbf{n}$ & $\mathbf{\%}$ \\
\hline Sexo & 55 & 57,9 \\
$\quad$ Feminino & 40 & 42,1 \\
$\quad$ Masculino & & \\
Idade & 72 & 75,8 \\
$\quad 60-79$ & 23 & 24,2 \\
$\quad 80$ ou mais & & \\
Situação conjugal & 50 & 52,6 \\
$\quad$ Sem companheiro & 45 & 47,4 \\
$\quad$ Com companheiro & & \\
Nível de escolaridade & 33 & 34.7 \\
$\quad$ Alfabetizado & 62 & 65,3 \\
Não Alfabetizado & & \\
*Renda mensal & 3 & 3,8 \\
$\quad$ Menos de 1 salário mínimo & 36 & 96,2 \\
$\quad \geq 1$ salário mínimo & 76 & \\
Etilismo & & 15,8 \\
$\quad$ Sim & 15 & 84,2 \\
$\quad$ Não & 80 & 19,5 \\
Tabagismo & & 80,5 \\
$\quad$ Sim & 17 & \\
Não & 70 & \\
\hline
\end{tabular}

*Salário mínimo na época (2011): R\$ 622,00

$\mathrm{Na}$ avaliação da capacidade funcional, observou-se que $3,4 \%(n=3)$ dos idosos eram dependentes para a realização das ABVD. Verificou-se associação entre atividade física e incapacidade funcional para a realização das ABVD na análise bruta. Quando estratificada por sexo e idade, a associação entre atividade física e incapacidade funcional não se manteve $(\mathrm{p}>0,05)$ (Tabela II).

A maioria dos idosos era ativa fisicamente $(64,2 \%$ $\mathrm{n}=61)$ e verificou-se associação estatisticamente significante entre nível de atividade física e idade $(\mathrm{p}=0,004)$, situação conjugal $(p=0,020)$ e escolaridade $(p=0,002)$ (Tabela III). 
Tabela II - Associação entre incapacidade funcional e nível de atividade física estratificada por sexo e idade da população idosa residente em zona rural estudada. Itajuru-BA, 2011.

\begin{tabular}{lcccc}
\hline Variáveis & \multicolumn{4}{c}{ Incapacidade Funcional } \\
Não sedentários & RP bruta & p valor & \\
Sedentários & 1 & 0,02 & Feminino & \\
& 1,09 & & $\mathbf{R P}$ & $\mathbf{p}$ valor \\
Masculino & & 1 & 0,189 \\
Não sedentários & $\mathbf{R P}$ & $\mathbf{p}$ valor & 1,10 & - \\
Sedentários & 1 & 0,316 & 80 anos ou mais & $\mathbf{p ~ v a l o r ~}$ \\
& 1,09 & - & $\mathbf{R P}$ & 0,293 \\
Não sedentários & $60-79$ anos & $\mathbf{p}$ valor & 1 & - \\
Sedentários & $\mathbf{R P}$ & 0.122 & 1,16 & \\
\hline
\end{tabular}

RP: Razões de prevalência

Tabela III - Nível de atividade física segundo características sociodemográficas e hábitos de vida de idosa residentes em zona rural estudada. Itajuru-BA, 2011.

\begin{tabular}{|c|c|c|c|}
\hline \multirow{2}{*}{ Variável } & \multicolumn{3}{|c|}{ Nível de Atividade Física } \\
\hline & Não sedentários (\%) & Sedentários (\%) & p valor \\
\hline \multicolumn{4}{|l|}{ Sexo } \\
\hline Masculino & 70,0 & 30,0 & 0,315 \\
\hline Feminino & 60,0 & 40,0 & \\
\hline \multicolumn{4}{|l|}{ Idade } \\
\hline 60-79 anos & 72,2 & 27,8 & 0,004 \\
\hline 80 ou mais & 39,1 & 60,9 & \\
\hline \multicolumn{4}{|l|}{ Situacão conjugal } \\
\hline Com companheiro & 75,6 & 24,4 & 0,020 \\
\hline Sem companheiro & 43,8 & 56,3 & \\
\hline \multicolumn{4}{|l|}{ Escolaridade } \\
\hline Não alfabetizado & 46,8 & 53,2 & 0,002 \\
\hline Alfabetizado & 15,2 & 84,8 & \\
\hline \multicolumn{4}{|l|}{$*$ Renda } \\
\hline Até um salário mínimo & 59,2 & 40,8 & 0,156 \\
\hline Z 1 salário mínimo & 100,0 & 0,0 & \\
\hline \multicolumn{4}{|l|}{ Etilismo } \\
\hline Não & 63,8 & 36,3 & 0,829 \\
\hline Sim & 66,7 & 33,3 & \\
\hline \multicolumn{4}{|l|}{ Tabagismo } \\
\hline Não & 65,7 & 34,3 & 0,595 \\
\hline Sim & 58,8 & 41,2 & \\
\hline
\end{tabular}

*Salário mínimo na época (2011): R\$ 622,00

\section{DISCUSSÃO}

Os resultados do presente estudo identificaram uma elevada frequência de indivíduos ativos fisicamente e uma baixa prevalência de dependência funcional. Mesmo com as limitações em relação ao desenho e ao tamanho da amostra do estudo, esses achados apontam para a importância da atividade física entre a população idosa e indica que esse comportamento (prática de atividade física habitual) deve fazer parte das estratégias de promoção à saúde.

O elevado percentual de idosos ativos fisicamente observados neste estudo contradizem os resultados de estudos conduzidos com idosos rurais do município de Uberaba-MG e idosos urbanos de 100 municípios brasileiros nos quais foi encontrada maior prevalência de sedentários ${ }^{(21,22)}$. 
As diferenças nesses achados podem ser justificadas pelas diferentes realidades e características dos municípios estudados. No caso específico da população desta pesquisa, foi observado que, mesmo após a aposentadoria, a maioria dos idosos continuava a desempenhar suas atividades no âmbito rural, caracterizado pelo cultivo de lavouras, deslocamento por distâncias longas em intensidades leve e moderadas até as fazendas, o que parece contribuir para a diminuição do sedentarismo entre os idosos investigados.

Além dos aspectos físicos, o estilo de vida ativo adotado pelos idosos praticantes de atividade física regular também exerce influência sobre a qualidade de vida, capacidade cognitiva, autoestima e integração social, possibilitando menor dependência, maior autonomia e sobrevivência desses idosos ${ }^{(23,24)}$.

Evidências indicam que os níveis de atividade física dos indivíduos tendem a diminuir com o passar dos anos ${ }^{(25,12)}$. Em estudo realizado com 124 idosos com idades entre 80 e $100 \operatorname{anos}^{(25)}$, foi constado que mais da metade dos idosos estudados não atendia às recomendações mínimas de prática de atividade física semanal. Dentre os principais motivos para a não adoção de um estilo de vida ativo pelos idosos, destacam-se as limitações por doenças, idade avançada e falta de motivação ${ }^{(26)}$. No presente estudo, a idade foi uma das variáveis associadas ao declínio do nível de atividade física entre os participantes. Nesse sentido, percebe-se que, com o passar dos anos, existe uma tendência de redução da prática de atividade física.

Outras características sociodemográficas podem influenciar o nível de atividade física, por exemplo: idosos sem companheiros e com maior escolaridade apresentam maiores níveis de atividade fisica ${ }^{(27,28)}$. Entretanto, no presente estudo, a maior predominância de atividade física foi observada entre os idosos com companheiros e com menor nível escolaridade.

Observou-se que a prevalência global de incapacidade funcional foi baixa, cenário que converge com resultados de estudos realizados em Santa Catarina e em Minas Gerais com idosos rurais ${ }^{(29,21)}$.

Os resultados encontrados no presente estudo são animadores, já que, com o avançar da idade, a capacidade funcional tende a declinar e pode atingir patamares indesejáveis, muitas vezes comprometendo a capacidade de realização de tarefas cotidianas ${ }^{(30)}$.

Ao analisar a associação entre atividade física e incapacidade funcional, identificou-se que a atividade física apresentou-se como um comportamento associado à capacidade funcional, apenas na análise bruta.

Achados da literatura reforçam a contribuição da prática regular de atividade física frente às atividades diárias (principalmente as ABVD). Um estudo ${ }^{(31)}$ demonstrou que
16 semanas de exercícios generalizados de intensidade moderada é um tempo suficiente para obter incrementos significativos na aptidão funcional (avaliado por meio da bateria da American Alliance for Health, Physical Education, Recreation and Dance (AAHPERD) - composta por cinco testes: coordenação, flexibilidade, resistência de força, agilidade e equilíbrio dinâmico e resistência aeróbia geral) de idosos. Outro estudo ${ }^{(32)}$ avaliou o efeito de dois protocolos no desempenho das atividades da vida diária em mulheres idosas: 1) exercícios com pesos (execução de três séries de oito a 12 repetições a $60 \%$ de uma repetição máxima no exercício leg press $45^{\circ}$ e 2) atividades aeróbias (pedalar em cicloergometro durante 40 minutos a $60 \%$ da frequência cardíaca de reserva) com frequência de três vezes por semana, durante um período de cinco semanas. Concluiu-se que tanto o exercício com pesos como o aeróbico induziram efeito positivo nas atividades da vida diária.

Observa-se que a prática regular de atividade física exerce efeitos positivos na prevenção e minimização dos efeitos deletérios do envelhecimento ${ }^{(33)}$. Nesse contexto, as estratégias de promoção da saúde, principalmente no âmbito da atenção primária à saúde, devem fomentar a prática de atividade física entre a população idosa, tendo em vista seus benefícios para a manutenção da capacidade de realização das atividades diárias.

Dentre as limitações deste estudo, pode-se destacar o desenho da pesquisa. Um estudo de corte transversal se limita a avaliar a causa e o efeito das variáveis. Em contrapartida, este estudo foi conduzido com uma população pouco estudada no Brasil, portanto, poderá servir como insumo para a elaboração de ações direcionadas a essa população.

Por fim, recomenda-se a realização de outros estudos com a população residente em zona rural, envolvendo populações maiores e com acompanhamento longitudinal, no intuito de ampliar o conhecimento acerca da associação entre atividade física e incapacidade funcional.

\section{CONCLUSÃO}

A prática regular de atividade física apresenta-se como um comportamento importante na prevenção/melhora da incapacidade funcional entre a população de idosos avaliados.

\section{REFERÊNCIAS}

1. Veras R. Envelhecimento populacional contemporâneo: demandas, desafios e inovações. Rev Saúde Pública. 2009;43(3):548-54.

2. Deschenes MR. Effects of aging on muscle fibre type and size. Sports Med. 2004; 34(12):809-24. 
3. Doherty TJ. Invited review: aging and sarcopenia. J Appl Physiol. 2003;95(4):1717-27.

4. Silva TAA, Frisoli Junior A, Pinheiro MM, Szejnfeld VL. Sarcopenia associada ao envelhecimento: aspectos etiológicos e opções terapêuticas. Rev Bras Reumatol. 2006;46(6):391-7.

5. Minosso JSM, Amendola F, Alvarenga MRM, Oliveira MAC. Prevalência de incapacidade funcional e dependência em idosos atendidos em um centro de saúde-escola da universidade de São Paulo. Cogitare Enferm. 2010;15(1):12-8.

6. Caldas CP. Envelhecimento com dependência: responsabilidades e demandas da família. Cad Saúde Pública. 2003;19(3):733-81.

7. Christensen U, Stovring N, Schultz-Larsen K, Schroll M, Avlund K. Functional ability at age 75: is there an impact of physical inactivity from middle age to early old age? Scand J Med Sci Sports. 2006;16(4):245-51.

8. Okuma SS. O idoso e a atividade física: fundamentos e pesquisa. $3^{\text {a }}$ ed. São Paulo: Papirus; 2004.

9. Virtuoso Junior JS, Tribess S, Paulo, TRS, Martins CA, Romo-Perez V. Atividade física como indicador preditivo para incapacidade funcional em pessoas idosas. Rev Latinoam Enferm. 2012;20(2):1-7.

10. Penha JCL, Piçarro IC, Barros Neto TL. Evolução da aptidão física e capacidade funcional de mulheres ativas acima de 50 anos de idade de acordo com a idade cronológica, na cidade de Santos. Ciênc Saúde Coletiva. 2012;17(1):245-53.

11. Matsudo SM. Atividade física na promoção da saúde e qualidade de vida no envelhecimento. Rev Bras Educ Fís Esp. 2006;20(Supl 5):135-7.

12. Hallal PC, Andersen LB, Bull FC, Guthold R, Haskell W, Ekelund U. Global physical activity levels: surveillance progress, pitfalls, and prospects. Lancet. 2012;380(9838):247-57.

13. Alencar NA, Aragão JCB, Ferreira MA, Dantas EHM Avaliação da qualidade de vida em idosas residentes em ambientes urbano e rural. Rio de Janeiro. Rev Bras Geriatr Gerontol. 2010;13(1):103-9.

14. Knuth AG, Bacchieri G, Victora CG, Hallal PC. Changes in physical activity among Brazilian adults over a 5-year period. J Epidemiol Community Health. 2010;64(7):591-5.

15. Knuth AG, Hallal PC. Temporal trends in physical activity: a systematic review. J Phys Act Health. 2009;6(5):548-59.
16. Ogilvie D, Foster CE, Rothnie H, Cavill N, Hamilton V, Fitzsimons $\mathrm{CF}$, et al. Interventions to promote walking: systematic review. BMJ. 2007;334:1204.

17. Instituto Brasileiro de Geografia e Estatística - IBGE. Censo demográfico. Características da população e dos domicílios: resultados do universo. Rio de Janeiro: IBGE; 2010.

18. Katz S, Stroud MW. Functional assessment in geriatrics: a review of progress and directions. J Am Geriatr Soc. 1989;37(3):267-71.

19. Benedetti TRB, Borges LJ, Petroski EL, Gonçalves LHT Atividade física e estado de saúde mental de idosos. Rev Saúde Pública. 2008;42 (2):302-7.

20. Tavares DMS, Arduini AB, Dias FA, Ferreira PCS, Oliveira EA. Perfil sociodemográfico, capacidade funcional e qualidade de vida de homens idosos residentes na zona rural. Rev de enferm e atenção à saúde. 2012;1(1):17-29.

21. Madeira MC, Siqueira FCV, Facchini LA, Silveira DS, Tomasi E, Thumé SSM, et al. Atividade física no deslocamento em adultos e idosos do Brasil: prevalências e fatores associados. Cad Saúde Pública. 2013;29(1):165-74.

22. Chaim J, Raimundo ME, Ferreira CAS, Yuaso DR. Prática regular de atividade física e sedentarismo: influência na qualidade de vida de idosas. Passo Fundo. RBCEH. 2010;7(2):198-209.

23. Rocha SV, Tribess S, Virtuoso Júnior JS. Atividade física habitual e qualidade de vida de mulheres idosas com baixa condição econômica. Rev Educação Física UEM. 2008; 19(1):101-8.

24. Andrade EL, Matsudo SMM, Matsudo VKR, Araújo TL, Andrade DR, Oliveira LC, et al. Barriers and motivational factors for physical activity adherence in elderly people in developing country [abstract]. Med Sci Sports Exerc. 2000;33(7):141.

25. Boscatto EC, Duarte MFS, Barbosa AR. Nível de atividade física e variáveis associadas em idosos longevos de Antônio Carlos, SC. Pelotas/RS. Rev Bras Ativ Fis Saúde. 2012;17(2):132-6.

26. Lopes MA, Krug RR, Mazo GZ, Bonetti M. Motivos de não adoção à prática de atividade física por pessoas longevas. Viçosa. Rev Mineira Educ Fís. 2012;1:114553.

27. Massa KHC, Guimarães VV, César CLG, Barros MBA, Carandina L, Goldbaum M, et al. Atividade física e hipertensão em idosos no Município de São Paulo. Pelotas/RS. Rev Bras Ativ Fis Saúde. 2012;17(1):7-13. 
28. Del Duca GF, Silva MC, Hallal PC. Incapacidade funcional para atividades básicas e instrumentais da vida diária em idosos. Rev Saúde Pública. 2009;43(5):796805.

29. Fhon JRS, Diniz MA, Leonardo KC, Kusumota L, Haas VJ, Rodrigues RAP. Síndrome de fragilidade relacionada à incapacidade funcional no idoso. Acta Paul Enferm. 2012;25(4):589-94.

30. Brown CJ, Flood KL. Mobility limitation in the older patient: a clinical review. J Am Med Assoc. 2013;310(11):1168-77.

31. Nascimento CMC, Ayan C, Cancela JM, Pereira JR, Andrade LP, Garuffi M, et al. Exercícios físicos generalizados capacidade funcional e sintomas depressivos em idosos brasileiros. Rev Bras Cineantropom Desempenho Hum. 2013;15(4):486-97.
32. Raso V, Grave JMD. Exercício aeróbico ou com pesos melhora o desempenho nas atividades da vida diária de mulheres idosas. Rev Bras Med Esporte. 2012;18(02):87-90.

33. Spirduso WW. Dimensões físicas do envelhecimento. São Paulo: Manole; 2005. p. 38-56.

\section{Endereço para correspondência:}

Saulo Vasconcelos Rocha

Universidade Estadual do Sudoeste da Bahia - UESB

Núcleo de Estudos em Saúde da População - NESP

Avenida José Moreira Sobrinho, $\mathrm{s} / \mathrm{n}$

Bairro: Jequiezinho

CEP: 45200-000 - Jequié - BA - Brasil

E-mail: svrocha@uesb.edu.br 